Research Article

\title{
Sensitivity, advantages, limitations, and clinical utility of targeted next-generation sequencing panels for the diagnosis of selected lysosomal storage disorders
}

\author{
Diana Rojas Málaga ${ }^{1,2}$, Ana Carolina Brusius-Facchin ${ }^{2}$, Marina Siebert ${ }^{3}$, Gabriela Pasqualim ${ }^{1,3,5}$ (D), Maria \\ Luiza Saraiva-Pereira ${ }^{1,2,6}$ iD, Carolina F.M de Souza ${ }^{2}$, Ida V.D. Schwartz ${ }^{1,2,4}$, Ursula Matte ${ }^{1,3,4}$ and Roberto \\ Giugliani $^{1,2,4}$ (iD) \\ ${ }^{1}$ Postgraduate Program in Genetics and Molecular Biology, Universidade Federal do Rio Grande do Sul, \\ Porto Alegre, RS, Brazil. \\ ${ }^{2}$ Medical Genetics Service, Hospital de Clinicas de Porto Alegre, Porto Alegre, RS, Brazil. \\ ${ }^{3}$ Experimental Research Center, Hospital de Clinicas de Porto Alegre, Porto Alegre, RS, Brazil. \\ ${ }^{4}$ Department of Genetics, Universidade Federal do Rio Grande do Sul, Porto Alegre, RS, Brazil. \\ ${ }^{5}$ Gene Therapy Center, Hospital de Clínicas de Porto Alegre, Ramiro Barcelos, 2350, Porto Alegre, RS, \\ Brazil. \\ ${ }^{6}$ Department of Biochemistry, Universidade Federal do Rio Grande do Sul, Porto Alegre, Brazil
}

\begin{abstract}
Lysosomal storage disorders (LSDs) constitute a heterogeneous group of approximately 50 genetic disorders. LSDs diagnosis is challenging due to variability in phenotype penetrance, similar clinical manifestations, and a high allelic heterogeneity. A powerful tool for the diagnosis of the disease could reduce the "diagnostic odyssey" for affected families, leading to an appropriate genetic counseling and a better outcome for current therapies, since enzyme replacement therapies have been approved in Brazil for Gaucher, Fabry, and Pompe diseases, and are under development for Niemann-Pick Type B. However, application of next-generation sequencing (NGS) technology in the clinical diagnostic setting requires a previous validation phase. Here, we assessed the application of this technology as a fast, accurate, and cost-effective method to determine genetic diagnosis in selected LSDs. We have designed two panels for testing simultaneously 11 genes known to harbor casual mutations of LSDs. A cohort of 58 patients was used to validate those two panels, and the clinical utility of these gene panels was tested in four novel cases. We report the assessment of a NGS approach as a new tool in the diagnosis of LSDs in our service.
\end{abstract}

Keywords: Ion Torrent, molecular diagnostics, next-generation sequencing, lysosomal storage disorders, validation.

Received: April 26, 2018; Accepted: October 18, 2018.

\section{Introduction}

Lysosomal storage disorders (LSDs) comprise a heterogeneous group of at least 50 rare genetic disorders caused by progressive accumulation of specific substrates, generally due to a deficiency of a lysosomal enzyme (Filocamo and Morrone, 2011). A main factor related to diagnosis delay is the wide spectrum of clinical manifestations of variable severity that are not specific of the disorder and can overlap with symptoms of other LSDs (Vieira et al., 2008; Martins et al., 2013). Another challenge is the high allelic heterogeneity for genetic screening. Early diagnosis is important since enzyme replacement and other available

Send correspondence to Ana Carolina Brusius Facchin. Medical Genetics Service, Hospital de Clinicas de Porto Alegre, Rua Ramiro Barcelos 2350, 90035-903 Porto Alegre, RS, Brazil. E-mail: afacchin@hcpa.edu.br. therapies improve the natural course of many of these diseases (Tajima et al., 2013; Muenzer, 2014; Franco et al., 2016; Giugliani et al., 2016).

The established approach to the diagnosis of patients with LSDs include the detection of the accumulated substrate whenever possible and the activity assay of the deficient enzyme, followed by Sanger sequencing of the gene associated with the disorder, which can be expensive and time consuming (Wang et al., 2011). Fortunately, new technologies are becoming more accessible and relatively affordable for the diagnostic routine. Targeted next-generation sequencing (TNGS) allows the simultaneous screening of several LSDs-related genes, with great depth of coverage, manageable interpretation, and relative low risk of finding variants of unknown significance, decreasing 
turnaround times for the final report (Rehm et al., 2013; Bhattacharjee et al., 2015).

However, before using TNGS technologies as a diagnostic tool, the validation of each test offered in the clinical setting is required. This validation is essential for stablishing critical parameters, from sample processing to analysis and interpretation steps, following the recommendations of published guidelines (Gargis et al., 2012; Rehm et al., 2013).

Here, we present the development and validation of two different TNGS panels of genes related to a subgroup of LSDs, offered as a diagnostic alternative by a Brazilian reference service for rare diseases. The sensitivity, advantages, drawbacks, and clinical utility of these TNGS panels are then reported.

\section{Subjects and Methods}

\section{Gene panel design}

Genes associated with LSDs with overlapping clinical manifestations, as well as related deficiencies were in- cluded in our panels (Figure 1). The two panels comprised 11 genes: Panel A: GLA (Fabry disease), NAGA (Schindler disease), GAA (Pompe disease), and LAMP2 (Danon disease), and Panel B: $N P C 1$ (Niemann-Pick disease type C1), NPC2 (Niemann-Pick disease type C2), GBA1 (Gaucher disease), LIPA (Lysosomal acid lipase deficiency), SMPD1 (Niemann-Pick disease type A/B), CHIT1 (Chitotriosidase deficiency), and PSAP (Prosaposin deficiency and saposin $B$ deficiency). Custom primers were designed using Ion Ampliseq $^{\mathrm{TM}}$ Designer v3.4 (Thermo Fisher Scientific) to generate a pool of primers for amplification of genomic regions of interest. Each one consists of two primer pools that target the entire coding region, including $20 \mathrm{bp}$ of intronexon junctions. Missed areas in the design were filled in by Sanger sequencing to reach a $100 \%$ breadth of coverage.

\section{Subjects}

The validation phase was performed using whole blood genomic DNA extracted by a standard saline extraction method (Miller et al., 1988), from 55 diagnosed patients (22 for panel A and 33 for panel B) who underwent
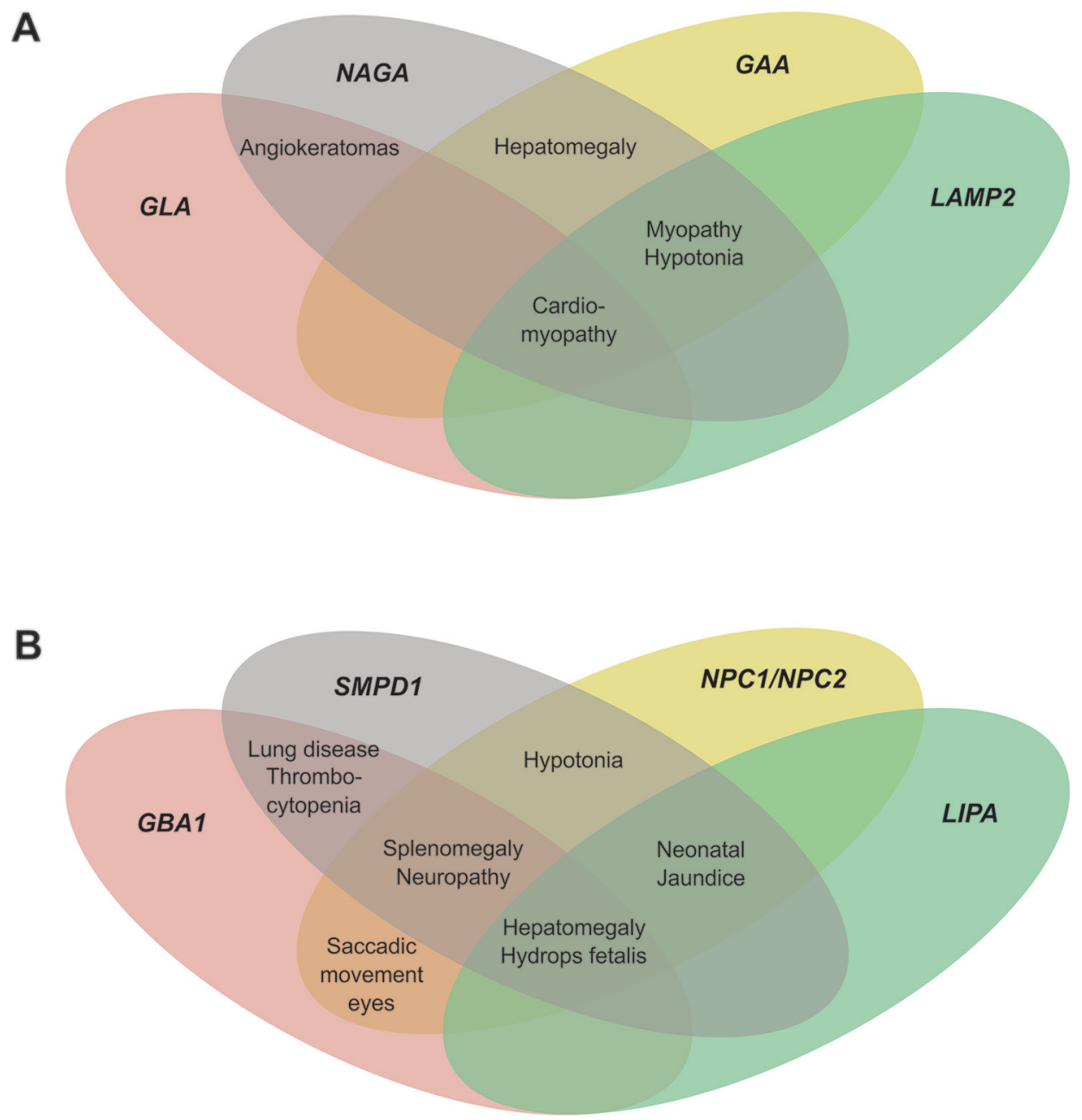

Figure 1 - Overlapping of clinical manifestations among LSDs. Venn's four-set diagram represented by causal genes. A, Panel A. B, Panel B. 
previous investigation with biochemical tests and Sanger sequencing (with known mutations and polymorphisms, including SNPs and small indels). Samples from three healthy adults were also analyzed. All probands were recruited from patients attended at the Medical Genetic Service, Hospital de Clinicas de Porto Alegre, Brazil. All samples were anonymized, sequenced, and analyzed in a single blind manner. TNGS was performed using an Ion Torrent Personal Genome Machine ${ }^{\mathrm{TM}}\left(\mathrm{PGM}^{\mathrm{TM}}\right)$ System (Thermo Fisher Scientific). The clinical utility of the validated tests was assessed by evaluating four patients with suspected LSDs. The study was approved by the institutional Ethics Committee of HCPA (\#15-0165).

\section{Multiplex PCR enrichment, library construction, and massive parallel sequencing}

The reagents used in these analyses were from Thermo Fisher Scientific, unless otherwise stated. Twenty nanograms of each gDNA sample were used for PCR enrichment of targets by applying the two custom Ampliseq $^{\text {TM }}$ panels. Each panel consisted of two separate PCR primer pools. The library was constructed using Ion AmpliSeq Library kit 2.0. Eight to nine samples barcoded with Ion Xpress Barcode Adapters kit were included in each set of library preparations. Unamplified libraries were purified with an Agencourt AMPure XP kit (Beckman Coulter). Libraries were prepared in equimolar concentrations using the Ion Library Equalizer kit, or quantified using the Qubit ${ }^{\mathbb{B}}$ dsDNA HS kit, followed by dilution to the same concentration. For template preparation, the barcoded libraries were pooled in equimolar concentrations of $100 \mathrm{pM}$ each and were subsequently submitted to emulsion PCR (emPCR) using the Ion PGM Template OT2 200 kit on the Ion OneTouch2 Instrument (Thermo Fisher Scientific). The percentage of positive Ion Sphere Particles (ISPs) was defined by flow cytometry performed on an Attune ${ }^{\circledR}$ Acoustic Focusing Flow Cytometer (Thermo Fisher Scientific) according to the demonstrated protocol (Part. no. 4477181). Positive ISPs were enriched using Ion OneTouch ES (Enrichment System).

All barcoded samples were loaded onto Ion $314^{\mathrm{TM}}$ chips v2 (Thermo Fisher Scientific) taking up to 8-9 samples on a single chip per sequencing run. Chip loading was performed according to the user guide for the Ion PGM sequencing 200 kit v2 (Thermo Fisher Scientific), following the manufacturer's instruction.

\section{Data analysis}

Raw signal data were analyzed using Torrent Suite Software v.5.0 (Thermo Fisher Scientific). Primary analyses included signal processing, base calling, demultiplexing, read alignment to human genome reference 19 (Genome Reference Consortium GRCh37), quality control of mapping quality, coverage analysis, and variant calling. Subsequently, a list of detected sequence variants, includ- ing SNPs and small insertions/deletions, was imported into Ion Reporter ${ }^{\mathrm{TM}}$ Software (Thermo Fisher Scientific) for annotation. Alignments were visually verified with the Integrative Genomics Viewer (IGV) v2.3 (Robinson et al., 2011).

Candidate variants met the following criteria: be detected on both strands and account for $20 \%$ of total reads at that site, quality score $\geq 20$, minimum read depth of $100 \mathrm{X}$ and variant frequency in the population $\leq 1 \%$. The filtered variants were then compared to mutation databases, including dbSNP (htpp://www.ncbi.nlm.nih.gov/projects/SNP/), 1000G (http://browser.1000genomes.org), ExAC (http://exac.broadinstitute.org), Online Archive of Brazilian Mutations (http://abraom.ib.usp.br/), HGMD (http://www.hgmd.cf.ac.uk/ac/), Pompe Center at Erasmus Medical Center (http://www.pompecenter.nl/), Fabry-database.org (http://fabry-database.org/), and the International Niemann-Pick Rare Disease Registry (https://inpdr.org/). All databases were last accessed in September 2017. Evaluation of the pathogenicity of the novel variants of unknown significance (VUS) (i.e., not found in any of the mutation databases, or not previously described in the literature) were analyzed with in silico web tools, such as SIFT (Kumar et al., 2009; Sim et al., 2012), Polyphen-2 (Adzhubei et al., 2010), and Mutation Taster (Schwarz et al., 2014), to predict potential protein deleterious effects on protein function. To evaluate the possible effect of synonymous variant in gene splicing, we used the Human Splicer Finding web tool (Desmet et al., 2009). Indels were analyzed by VEST (Variant Effect Scoring Tool), VEP (Vep Effect Predictor), as well as Mutation Taster (Carter et al., 2013; Douville et al., 2016; McLaren et al., 2016). Nonsense, frameshift, and canonical splice mutations were classified automatically as pathogenic (Richards et al., 2015).

\section{Performance characteristics}

Run metrics and coverage analyses were performed to identify systematic deficiencies. We analyzed depth of coverage (DoC) in the targeted amplicons to assess target enrichment across all 58 samples data sets and establish an acceptable reference range for key measures.

Two coverage analyses were generated: (1) Highlevel DoC overview plot based on Tayoun et al. (2013), with relative $\mathrm{DoC}$ in the $\mathrm{y}$-axis and amplicons on the $\mathrm{x}$-axis, highlighting in red the amplicons with significantly lower coverage (Figure 2); and (2) relative DoC plot of exons per gene (Supplementary Figures S1 and S2). Direct visual inspection of amplicon reads on IGV v2.3 and evaluation of high-level DoC coverage overview were used to establish the reportable ranges for each panel.

Sensitivity and specificity were calculated (overall and for each gene) and compared with results obtained by standard Sanger sequencing. False negative and positive overall rates were also calculated. To assess reproducibility 
A

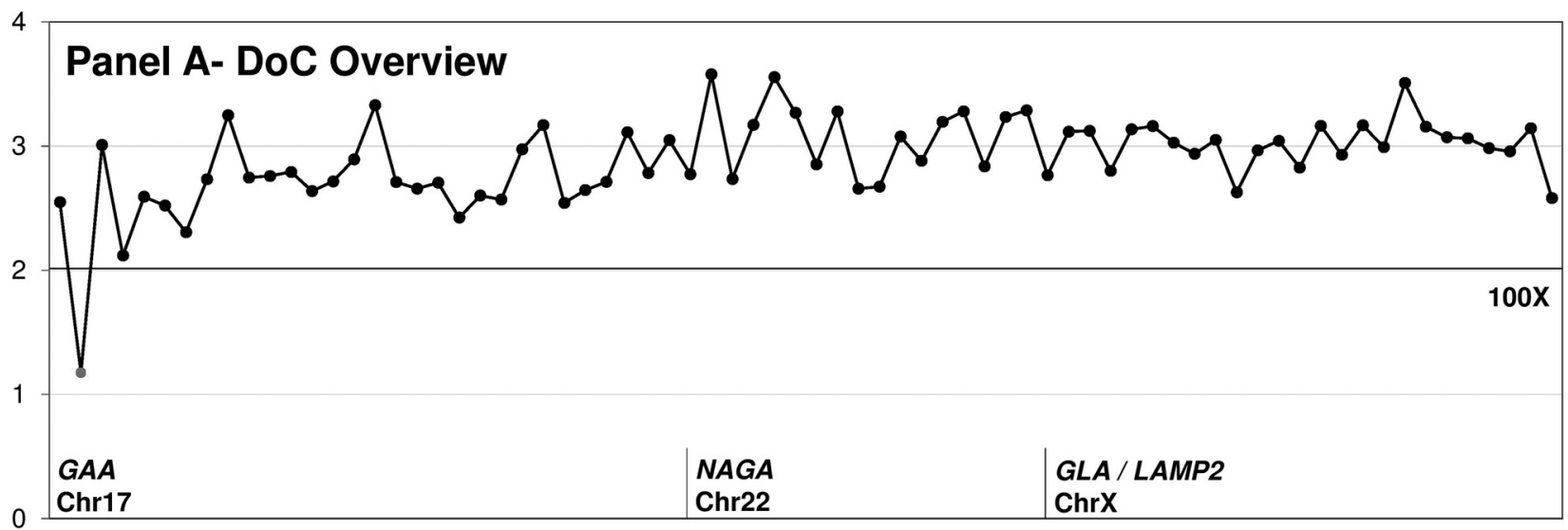

B

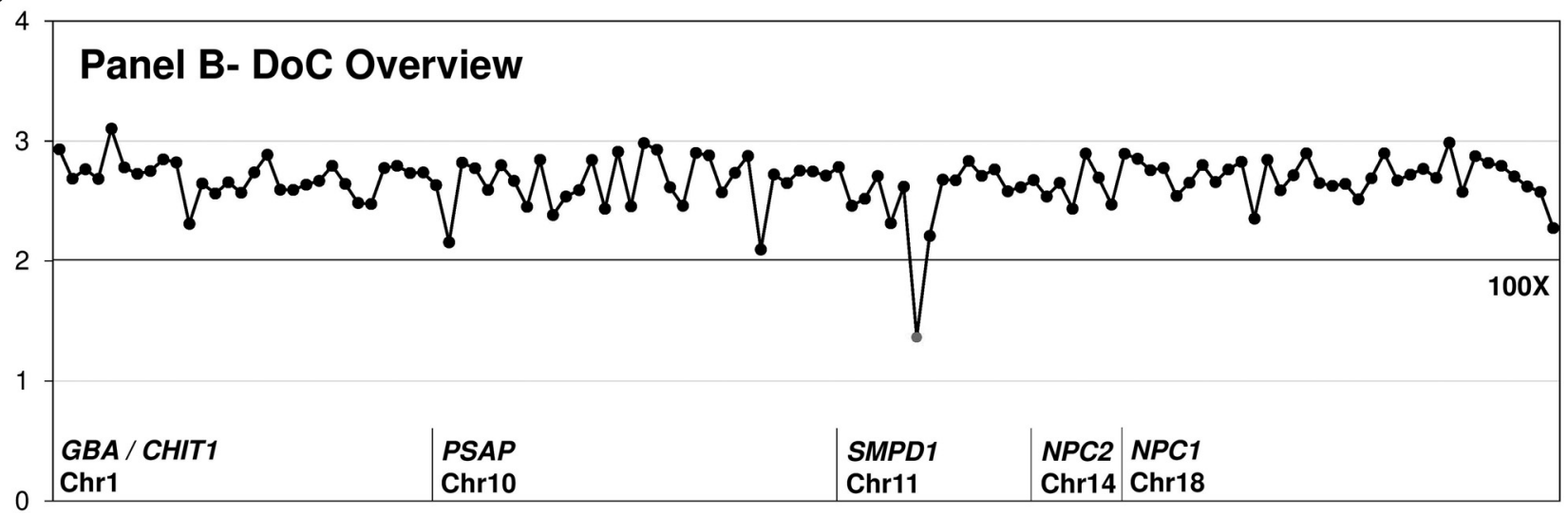

Figure 2 - Depth of Coverage (DoC) of panels presented in this study. Overview of all 191 custom amplicons designed for Panel A (73 amplicons) (upper panel A) and Panel B (118 amplicons) (lower panel B) for TNGS. The line indicates a DoC of 100X. Relative DoC is on the y-axis and amplicons are on the $\mathrm{x}$-axis.

of the assay, we measured concordance between independent runs using relative DoC.

\section{Sanger sequencing}

Sanger sequencing was performed for confirmation of all variants, to fill the regions missed by the custom panel design and low-coverage regions, and for the analysis of clinical utility. gDNA was amplified using specific primers designed for the free software Primer3 v.0.4.0. (available upon request). Amplicons were sequenced by both ends using the Big Dye Terminator v3.1 cycle sequencing kit (Thermo Fisher Scientific), and fragments were resolved on an ABI 3500 DNA Analyzer (Thermo Fisher Scientific). Analysis of results was performed with the BioEdit v7.2.5 free software.

Table 1 - Performance characteristic for both LSDs panels
Results

\section{Run metrics}

Our designs generated a total of 73 and 118 amplicons for Panel A and B, respectively. Mean amplicon read length was 150-180 bp. Sequencing of genes generated reads in the range of 69,000 to 7,600 per sample. An evenly distributed mean depth of coverage for both panels was achieved and a mean of $95 \%$ targeted bases were covered at least $100 \mathrm{X}$. The other run metrics are summarized in Table 1.

\section{Coverage analysis}

An overview on coverage for all analyzed samples is shown in Figure 1. Although the coverage for GAA and $S M P D 1$ was expected to be 94.54 and $100 \%$, respectively,

\begin{tabular}{ccccccccc}
\hline Panel & Breadth of coverage & Mapped reads per sample & \multirow{2}{*}{ On target } & \multirow{2}{*}{ Mean depth (X) Uniformity } & \multicolumn{3}{c}{ \% Target bases covered } \\
\cline { 5 - 8 } & & & & & \multicolumn{2}{c}{ 20x } & $100 x$ & $500 x$ \\
\hline A & $97.74 \%$ & $76,729 \pm 31195$ & $0.95 \pm 0.02$ & $812 \pm 339$ & $0.91 \pm 0.06$ & $98.66 \pm 0.49$ & $95.55 \pm 2.17$ & $55.06 \pm 18.08$ \\
B & $99.67 \%$ & $69,044 \pm 26566$ & $0.86 \pm 0.05$ & $498 \pm 198$ & $0.98 \pm 0.01$ & $99.72 \pm 0.14$ & $94.71 \pm 6.41$ & $56.42 \pm 25.39$ \\
\hline
\end{tabular}


the actual mean coverage was found to be 92.46 and $97.22 \%$. The coverage analysis demonstrated two regions poorly covered in these genes, as shown in more detail in Figures S1 and S2. Unfortunately, the low covered region contained the location of the c.573delT (p.Ser192fs) SMPD1 mutation. The actual coverage for all the other genes was as expected to be $100 \%$ based on probe design.

\section{Sensitivity}

To assess the analytical sensitivity of the panels (Tables 2 and 3), we compared the results obtained by Sanger sequencing to those obtained by TNGS, including as many different types of variations as possible: nonsense, missense, small deletions, small insertions, splicing, and intronic variants. A total of 57 variants (pathogenic and polymorphisms) were analyzed (Table 4). We also identified their correct zygosity status (data not shown).

Our assay identified precisely all recurrent mutations for LSDs, except two in Panel B. For this panel, the limitations were the inability to detect (1) SMPD1 c.573delT, p.Ser192fs, located in a region with low coverage, and (2) GBA1 c.[1448T > G; $1483 \mathrm{G}>\mathrm{C} ; 1497 \mathrm{G}>\mathrm{C}]$, p.[Leu444Pro;Ala456Pro,Val460Val] (Tables 2 and 4).

\section{Specificity}

Of all sequenced DNA samples, we identified threee false positives in panel A ( 3 in 7476 true negatives) and five false positives in panel B (5 in 15,054 true negatives), resulting in a specificity value of $99.96 \%$ $(95 \% \mathrm{CI}=0.998-0.999) \quad$ and $\quad 99.97 \%$ (95\%CI $=0.9992-0.999)$, respectively (Table 2 ). Specificity by gene is shown in Table 3 . These false positives were located in low coverage regions, which are prone to sequencing errors.

\section{Reproducibility}

To determine the reproducibility of our assay, we sequenced 24 samples divided in three independent runs for Panel A, and 34 samples divided in four independent runs for Panel B (Figure 3).

\section{Clinical Utility Assessment}

\section{Case 1}

A 15 -year-old male patient with suspicion of having a LSD was referred to our service via NPC Brazil Network. The main clinical findings were unexplained hepatosplenomegaly and myelogram with presence of numerous his-
Table 3 - Analytical sensitivity and specificity for each gene in our gene panels.

\begin{tabular}{lccc}
\hline Panel & Gene & Sensitivity & Specificity \\
\hline $\mathrm{A}$ & GLA & $100 \%(10 / 10)$ & $100 \%(1398 / 1398)$ \\
& NAGA & $100 \%(3 / 3)$ & $100 \%(1381 / 1381)$ \\
& GAA & $100 \%(12 / 12)$ & $99.89 \%(2987 / 2990)$ \\
& LAMP2 & n.d* & $100 \%(1707 / 1707)$ \\
\hline $\mathrm{B}$ & NPC1 & $100 \%(8 / 8)$ & $100 \%(4265 / 4265)$ \\
& NPC2 & $100 \%(1 / 1)$ & $100 \%(5521 / 5521)$ \\
& GBA1 & $83.3 \%(5 / 6)$ & $100 \%(1875 / 1875)$ \\
& LIPA & $100 \%(4 / 4)$ & $100 \%(1462 / 1462)$ \\
& SMPD1 & $91.7 \%(11 / 12)$ & $99.8 \%(2775 / 2780)$ \\
& CHIT1 & $100 \%(1 / 1)$ & $100 \%(1936 / 1936)$ \\
& PSAP & n.d* & $100 \%(2184 / 2184)$ \\
\hline
\end{tabular}

n. $d^{*}$ : not determined because there were no positive controls with variant pathogenic in this gene.

tiocytes. Several biochemical assays were performed to reach a diagnosis, including measurement of oxysterol and activity of chitotriosidase, lysosomal acid lipase, and bgalactosidase as reference enzymes that were all within normal ranges. Filipin test was inconclusive. Eventually, NPA/B was suspected and ASM enzyme activity was tested in cultured skin fibroblasts, resulting in $1.25 \mathrm{nmol} / \mathrm{h} / \mathrm{mg}$ protein (reference value: 49-72), indicating NPA/B disease. Due to several factors, like request of new samples for the biochemical assays, it took approximately 12 months to reach this biochemical diagnosis. Panel B, which includes genes related to LSD with hepatosplenomegaly as common clinical manifestation, was utilized as second-tier diagnostic approach. We found two pathogenic variants in SMPD1, both confirmed by Sanger sequencing: p.Arg610del (c.1826_1828delGCC) (rs120074118) and p.Asp420fs (c.1259delA), the latter being a novel, unreported mutation and not found in controls $(\mathrm{n}=32)$.

\section{Case 2}

A 21-month-old patient, daughter of consanguineous parents who presented macrocephaly and hepatosplenomegaly as main clinical features, high cholesterol (228 $\mathrm{mg} / \mathrm{dL}$ ) and triglycerides (492 mg/dL) levels, elevated liver enzymes (GGT: 137 IU/L; TGP: $256 \mathrm{IU} / \mathrm{L}$ ) as well as low levels of sphingomyelinase activity, was referred for molecular analysis of SMPD1 gene. TNGS (Panel B) revealed the homozygous small deletion p.Leu474fs

Table 2 - Analytical sensitivity, specificity, FN and FP rates for both TNGS panels.

\begin{tabular}{lcccccc}
\hline Panel & Pathogenic variants & $\begin{array}{c}\text { Polymorphism } \\
\text { NGS/Sanger }\end{array}$ & Sensitivity & Specificity & FN rate & FP rate \\
\hline A & $17 / 17$ & $8 / 8$ & $100 \%(25 / 25)^{*}$ & $99.96 \%$ & $0.000 \%$ & $0.040 \%$ \\
B & $20 / 22$ & $10 / 10$ & $93.75 \%(30 / 32)^{* *}$ & $99.97 \%$ & $0.063 \%$ & $0.033 \%$ \\
\hline
\end{tabular}

$* 95 \% \mathrm{CI}=0.875-1 ; * * 95 \% \mathrm{CI}=0.8091-0.9837, \mathrm{FN}$ : false negative, FP: false positive 
Table 4 - Variants detected in this study by TNGS and Sanger sequencing.

\begin{tabular}{|c|c|c|c|c|c|c|c|}
\hline Gene & Sequence reference & Location & cDNA change & Protein change & $\mathrm{dbSNP}$ & Mutation type & NGS detected \\
\hline \multirow[t]{10}{*}{ GLA } & NM_000169 & Exon 01 & c. $32 \mathrm{delG}$ & p.Gly11fs & - & Deletion & Yes \\
\hline & & Exon 01 & c. $4 \mathrm{C}>\mathrm{T}$ & p.Gln2Ter & - & Nonsense & Yes \\
\hline & & Exon 01 & c. $167 \mathrm{G}>\mathrm{A}$ & p.Cys56Tyr & - & Missense & Yes \\
\hline & & Exon 02 & c. $334 \mathrm{C}>\mathrm{T}$ & p.Arg112Cys & rs104894834 & Missense & Yes \\
\hline & & Exon 03 & c. $456 \mathrm{C}>\mathrm{A}$ & p.Tyr152Ter & - & Nonsense & Yes \\
\hline & & Exon 04 & c. $605 \mathrm{G}>\mathrm{A}$ & p.Cys202Tyr & rs869312344 & Missense & Yes \\
\hline & & Exon 05 & c. $644 \mathrm{~A}>\mathrm{G}$ & p.Asn215Ser & rs28935197 & Missense & Yes \\
\hline & & Exon 05 & c. $776 \mathrm{C}>\mathrm{G}$ & p.Pro259Arg & - & Missense & Yes \\
\hline & & Exon 05 & c. $790 \mathrm{G}>\mathrm{T}$ & p.Asp264Tyr & rs 190347120 & Missense & Yes \\
\hline & & Exon 07 & c. $1102 \mathrm{G}>\mathrm{A}$ & p.Ala368Thr & rs144994244 & Missense & Yes \\
\hline \multirow[t]{3}{*}{$N A G A$} & NM_000262.2 & Exon 03 & c. $279 \mathrm{G}>\mathrm{A}$ & p.Pro93Pro & rs133369 & Missense & Yes \\
\hline & & Exon 06 & c. $720 \mathrm{G}>\mathrm{A}$ & p.Gln240Gln & - & Missense & Yes \\
\hline & & Exon 08 & c. $973 \mathrm{G}>\mathrm{A}$ & p.Glu325Lys & rs121434529 & Missense & Yes \\
\hline \multirow[t]{12}{*}{$G A A$} & NM_001079804 & Intron 01 & c. $-32-13 \mathrm{~T}>\mathrm{G}$ & - & rs 386834236 & Splicing & Yes \\
\hline & & Exon 03 & c. $596 \mathrm{~A}>\mathrm{G}$ & p.His199Arg & rs1042393 & Missense & Yes \\
\hline & & Exon 03 & c. $668 \mathrm{G}>\mathrm{A}$ & p.Arg223His & rs 1042395 & Missense & Yes \\
\hline & & Intron 8 & c. $1327-18 \mathrm{~A}>\mathrm{G}$ & - & rs2278619 & Intron variant & Yes \\
\hline & & Exon 09 & c. $1374 \mathrm{C}>\mathrm{T}$ & p.Tyr458Tyr & rs1800305 & Missense & Yes \\
\hline & & Exon 10 & c. $1465 \mathrm{G}>\mathrm{A}$ & p.Asp489Asn & rs398123169 & Missense & Yes \\
\hline & & Exon 10 & c. $1504 \mathrm{~A}>\mathrm{G}$ & p.Met502Val & rs376067362 & Missense & Yes \\
\hline & & Exon 14 & c. $1905 \mathrm{C}>\mathrm{A}$ & p.Asn635Lys & - & Missense & Yes \\
\hline & & Exon 14 & c. $1941 \mathrm{C}>\mathrm{G}$ & p.Cys647Trp & - & Missense & Yes \\
\hline & & Intron 14 & c. $2040+20 \mathrm{~A}>\mathrm{G}$ & - & rs2304836 & Intron variant & Yes \\
\hline & & Exon 15 & c. $2065 \mathrm{G}>\mathrm{A}$ & p.Glu689Lys & rs1800309 & Missense & Yes \\
\hline & & Exon 18 & c. $2560 \mathrm{C}>\mathrm{T}$ & p.Arg854Ter & rs121907943 & Nonsense & Yes \\
\hline \multirow[t]{8}{*}{$N P C 1$} & NM_000271.4 & Exon 02 & $\begin{array}{c}\text { c.114_122del } \\
\text { GAGGTACAA }\end{array}$ & p.Lys38_Tyr40del & - & Deletion & Yes \\
\hline & & Exon 05 & c. $530 \mathrm{G}>\mathrm{A}$ & p.Cys177Tyr & rs 80358252 & Missense & Yes \\
\hline & & $\begin{array}{l}\text { Exon } \\
5,8,12\end{array}$ & $\begin{array}{c}\text { c. }[547 \mathrm{G}>\mathrm{A} ; 1093 \mathrm{~T}> \\
\mathrm{C} ; 1937 \mathrm{G}>\mathrm{A}]\end{array}$ & $\begin{array}{c}\text { p.[Ala183Thr;Ser365Pro; } \\
\text { Arg646His] }\end{array}$ & $\begin{array}{l}\text { rs111256741, -, } \\
\quad \text { rs112387560 }\end{array}$ & Missense & Yes \\
\hline & & Exon 20 & c. $3019 \mathrm{C}>\mathrm{G}$ & p.Pro1007Ala & rs80358257 & Missense & Yes \\
\hline & & Exon 21 & c. $3104 \mathrm{C}>\mathrm{T}$ & p.Ala1035Val & rs 28942107 & Missense & Yes \\
\hline & & Exon 21 & c. $3182 \mathrm{~T}>\mathrm{C}$ & p.Ile1061Thr & rs80358259 & Missense & Yes \\
\hline & & Intron 22 & c. $3477+3$ insCA & - & - & Insertion & Yes $^{+}$ \\
\hline & & Exon 24 & c.3662_3662delT & p.Phe1211fs & - & Deletion & Yes \\
\hline$N P C 2$ & NM_006432 & Exon 01 & c. $58 \mathrm{G}>\mathrm{T}$ & p.Glu20Ter & rs 80358260 & Nonsense & Yes \\
\hline \multirow[t]{6}{*}{$G B A 1$} & NM_001005742 & Exon 07 & c. $850 \mathrm{C}>\mathrm{A}$ & p.Pro245Thr & - & Missense & Yes \\
\hline & & Exon 07 & c.982_983insTGC & p.Leu327dup & rs121908298 & & Yes \\
\hline & & Exon 09 & c. $1226 \mathrm{~A}>\mathrm{G}$ & p.Asn370Ser & rs76763715 & Missense & Yes \\
\hline & & Exon 09 & c. $1251 \mathrm{G}>\mathrm{C}$ & p.Trp378Cys & - & Missense & Yes \\
\hline & & Exon 10 & c. $1448 \mathrm{~T}>\mathrm{G}$ & p.Leu444Pro & rs421016 & Missense & Yes \\
\hline & & Exon 10 & $\begin{array}{c}\text { c. }[1448 \mathrm{~T}>\mathrm{G} ; 1483 \mathrm{G}> \\
\mathrm{C} ; 1497 \mathrm{G}>\mathrm{C}]\end{array}$ & $\begin{array}{c}\text { p.[Leu444Pro; } \\
\text { Ala456Pro; Val460Val] }\end{array}$ & - & Missense & No \\
\hline \multirow[t]{4}{*}{$L I P A$} & NM_001127605 & Exon 02 & c. $67 \mathrm{G}>\mathrm{A}$ & p.Gly23Arg & rs1051339 & Missense & Yes \\
\hline & & Exon 08 & c. $894 \mathrm{G}>\mathrm{A}$ & p.Glu298Glu & rs116928232 & Missense & Yes \\
\hline & & Exon 10 & c. $1204 \mathrm{G}>\mathrm{A}$ & p.Gly342Arg & - & Missense & Yes \\
\hline & & Intron 05 & c. $539-5 \mathrm{C}>\mathrm{T}$ & - & rs2297472 & Intron variant & Yes \\
\hline
\end{tabular}




\begin{tabular}{|c|c|c|c|c|c|c|c|}
\hline \multirow[t]{12}{*}{$S M P D 1$} & NM_000543 & Exon 01 & c. $107 \mathrm{~T}>\mathrm{C}$ & p.Val36Val & rs1050228 & Missense & Yes \\
\hline & & Exon 02 & c. $338 \mathrm{G}>\mathrm{A}$ & p.Arg113His & rs149770879 & Missense & Yes \\
\hline & & Exon 02 & c. $573 \mathrm{delT}$ & p.Ser192fs & rs727504167 & Deletion & No \\
\hline & & Exon 02 & c. $636 \mathrm{~T}>\mathrm{C}$ & p.Asp212Asp & rs7951904 & Missense & Yes \\
\hline & & Exon 02 & c. $690 \mathrm{C}>\mathrm{G}$ & p.Arg230Arg & - & Missense & Yes \\
\hline & & Exon 02 & c. $714 \mathrm{~A}>\mathrm{G}$ & p.Ala238Ala & rs2682091 & Missense & Yes \\
\hline & & Exon 02 & c. $739 \mathrm{G}>\mathrm{A}$ & p.Gly247Ser & rs587779408 & Missense & Yes \\
\hline & & Exon 06 & c. $1522 \mathrm{GC}$ & p.Gly508Arg & rs1050239 & Missense & Yes \\
\hline & & Exon 06 & c. $1749 \mathrm{G}>\mathrm{A}$ & p.Ser583Ser & rs35098198 & Missense & Yes \\
\hline & & Exon 06 & c. $1805 \mathrm{G}>\mathrm{C}$ & p.Arg602Pro & - & Missense & Yes \\
\hline & & Exon 06 & c. $1805 \mathrm{G}>\mathrm{A}$ & p.Arg602His & rs370129081 & Missense & Yes \\
\hline & & Exon 06 & c.1826_1828delGCC & p.Arg608del & rs120074118 & Deletion & Yes \\
\hline CHIT1 & NM_003465.2 & Exon 04 & c. $304 \mathrm{G}>\mathrm{A}$ & p.Gly102Ser & rs2297950 & Missense & Yes \\
\hline
\end{tabular}

+: new mutation, confirmed by Sanger sequencing.
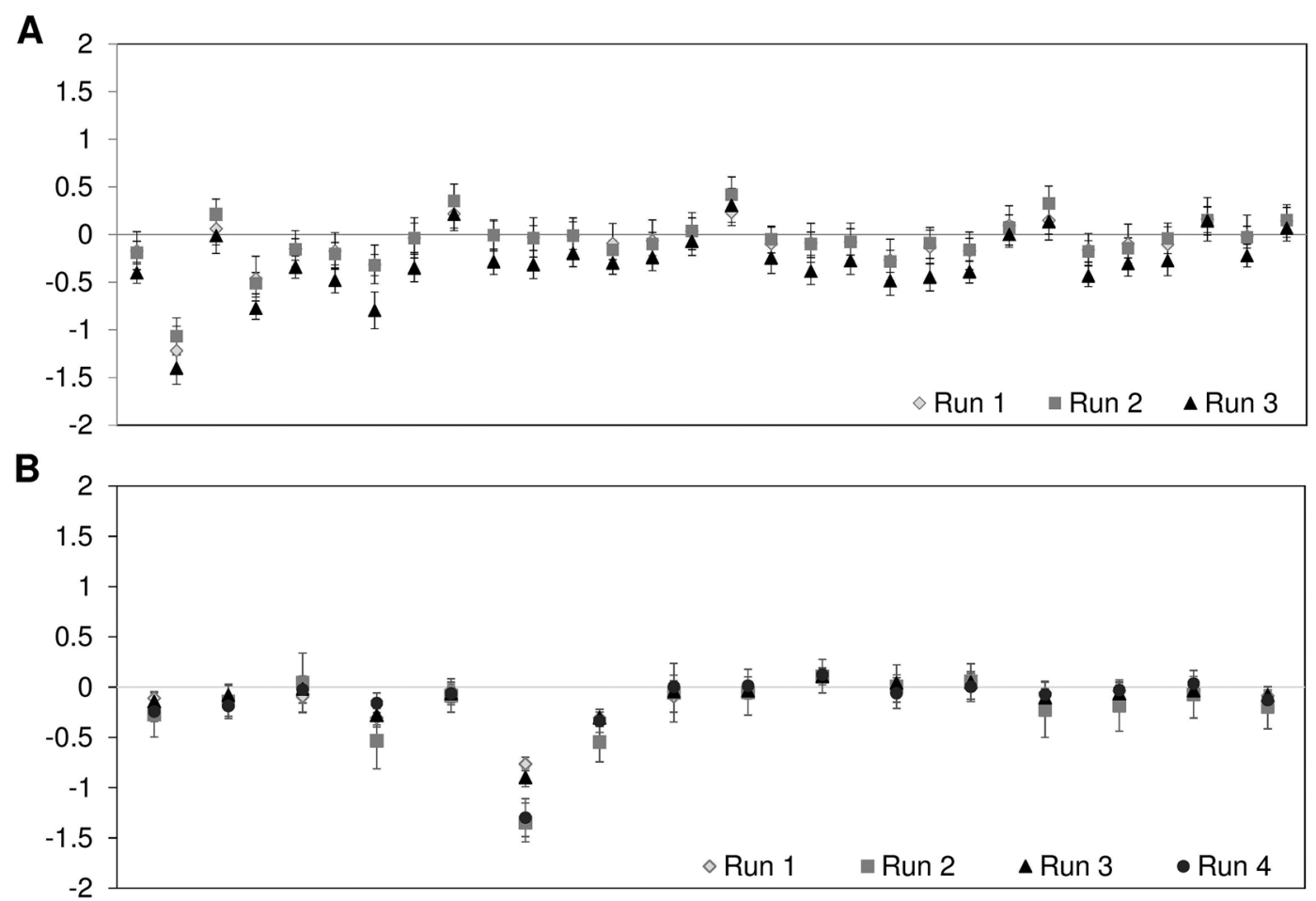

Figure 3 - Reproducibility of assays. (A) Mean relative DoC at 30 amplicons ( $G A A$ gene) of 8 different samples sequenced in 3 different assay runs; (B) Mean relative DoC at 16 amplicons (SMPD1 gene) of 8-9 different samples sequenced in 4 different assay runs. Error bars represent standard deviation.

(c.1420_1421delCT), which was reported previously as being pathogenic (rs398123476).

\section{Case 3}

A 21-year-old female, child of a non-consanguineous marriage, with diagnosis of hypertrophic cardiomyopathy at 18 years of age and with previous diagnosis of Danon disease, was referred to our service for mutation analysis of the LAMP2 gene. TNGS (Panel A) detected the hemi- zygous variant p.Asn242fs (c. 725delA), a novel pathogenic variant.

\section{Case 4}

A 16-year-old male with suspicion of Danon disease due to hypertrophic cardiomyopathy with anomalous pathway, presented intellectual deficiency, proximal myopathy, and alterations in liver tests. As in case 3, Panel A was used, detecting the hemizygous variant c. $741+1 \mathrm{G}>\mathrm{A}$, described as pathogenic (HGMD CS003703). 


\section{Discussion}

Due to various reasons, such as wide clinical and genetic heterogeneity, LSDs are difficult to diagnose, and it can take several years to reach a final diagnosis (Vieira et al., 2008; Martins et al., 2013). Even if no treatment is available for many of these disorders, genetic diagnosis has potential benefits, such as predicting the prognosis,and allowing genetic counselling and family screening. Recent studies highlight the clinical utility of TNGS technology for genetic diagnosis of LSDs (Wood et al., 2013; Fernandez-Marmiesse et al., 2014; Lévesque et al., 2016). Although there are some drawbacks to TNGS, such as the inability to detect large indels and structural variants, there are several advantages in applying this approach early in the investigation of patients with LSDs, like high coverage, completeness, low rate of incidental finding, and the potential to reduce the diagnostic delay. TNGS assays involve various technical steps, starting from sample preparation to analysis and data interpretation, and each one requires full validation. We presented data on the development and validation of two gene panels, designed following the criteria of overlapping clinical manifestations, to be offered as a diagnostics option by a reference center of rare diseases (Figure 1). Prior to TNGS, an enrichment step of the genes included in the panel is necessary through capture approaches based on hybridization or PCR-based strategies. The latter one is especially suitable for the investigation of regions with less than $100 \mathrm{~kb}$, has versatile design, and is the most convenient for analysis of genes with pseudogenes due to its high specificity, sensitivity, and reproducibility (Claes and De Leener, 2014). Despite some reported disadvantages for this approach (time-consuming, uneven coverage of the target regions due to unequal PCR efficiency across the various amplicons, allelic dropout, and difficulties to detect large deletion/insertion events), Ion Ampliseq targeted technology utilizes a PCR-based method (high throughput multiplex PCR) for this purpose, overcoming some of the limitations and providing high specificity (here, $99.96 \%$ and $99.97 \%$ ) and uniformity (91\%-98\% for both panels).

From the run metrics results, we can conclude that all samples were uniformly covered at depths that exceed the minimum coverage required $(100 \mathrm{X})$ for accurate calling of variants. The bioinformatics pipeline applied here demonstrated high sensitivity for Panel A (sensitivity $100 \%$ ) and Panel B (sensitivity 93.75\%) (Table 1). The use of normal controls $(n=3)$ allowed the identification of eight platformspecific false positive variants, which were filtered from subsequent analyses. A high reproducibility was observed revealing a high concordance between independent runs.

Inadequate coverage regions were identified by coverage plots (Figure 2), and these regions werfe completed by Sanger sequencing. A technical difficulty encountered was related to enrichment of some targets and, as a consequence, low sequence coverage was found. This was ob- served at two targets corresponding to the GAA and SMPD1 genes. A high GC-content region (70\%) was probably the main reason why the GAA amplicon was poorly covered $(\sim 20 \mathrm{X})$. For SMPD1, a low-covered amplicon $(\sim 30 \mathrm{X})$ was identified, showing both a GC-content of $66 \%$ and a homopolymeric region within the target. These are well-recognized limitations of NGS sequencing. As recommended by the American College of Medical Genetics, both tests achieved a $100 \%$ breadth of coverage when complemented with gold-standard DNA sequencing that improves clinical sensitivity.

Another major limitation of Panel B was the inability to detect the RecNciI allele, c.[1448T > G; $1483 \mathrm{G}>\mathrm{C}$; $1497 \mathrm{G}>$ C]/ p.[Leu444Pro;Ala456Pro,Val460Val]. High sequence similarity between functional genes and their pseudogenes can make the detection of genuine mutation difficult due to the ambivalent mapping in the analysis of NGS data, which cannot always be avoided. Sanger sequencing is generally used to elucidate the correct variant mapping (Claes and De Leener,2014). In our study, the presence of GBAP1, a highly homologous GBA1 pseudogene, complicated sequencing analysis by NGS, with the RecNciI allele being particularly difficult to assess since mutant bases in GBAl (exon 10) are the wild type sequence in its pseudogene. Panel B failed to detect this allele, representing a case of false negative when present, because variant-containing reads align to homologous loci. Our strategy to infer the presence of RecNciI was based on the employment of a global alignment strategy, analyzing the DoC of exon $10 G B A 1$ and the homologous GBAP1 region. We observed that in the presence of the Rec allele an uneven reads distribution was observed due to the exclusive alignment of variant-containing reads with GBAP1. As examples: (a) for homozygous N370S, we observed a DoC of $369 \mathrm{X}$ for GBA1 exon 10 and $370 \mathrm{X}$ for GBAP1 homologous region; (b) in the case of compound heterozygosity (N370S/RecNciI) for exon 10 GBA1, a DoC of 144X and $313 \mathrm{X}$ for $G B A P 1$ was observed. Therefore, the presence of this Rec allele was inferred, but Sanger sequencing using a specific primer pair for exon 10 was required for confirmation of this inference.

Clinical utility assessment was performed. Two pathogenic variants were found, SMPD1 p.Asp420fs (c.1259delA) and LAMP2 p.Asn242fs (c. 725delA), demonstrating that our TNGS panel is a sensitive tool, with faster turnaround times for provision of results, and relative low cost ( USD 320 per sample) when compared with Sanger sequencing of individual genes, and showing the potential role for diagnosis of LSDs in our Medical Genetics Service.

In conclusion, TNGS technology can be used for the simultaneous testing of a broad range of SNPs and indels, being a fast, accurate, and cost effective method for the diagnosis of selected LSDs. It allows faster diagnosis and earlier treatment of patients, contributing to reduce the mor- 
bidity of the diseases and improve patient survival and quality of life.

\section{Acknowledgments}

This work was funded by the FIPE/HCPA (Project number: 15-0165). DRM has a fellowship from Innovate Peru. The investigation was partially funded by INAGEMP (CNpq/ Coordenação de Aperfeiçoamento de Pessoal de Nível Superior - Brasil (CAPES) - Finance Code 001CAPES/FAPERGS).

\section{Conflict of interest}

The authors have no relevant affiliations or financial involvement with any organization or entity with a financial interest or financial conflict with the subject matter or materials discussed in the manuscript. This includes employment, consultancies, honoraria, stock ownership or options, expert testimony, grants or patents received or pending, or royalties.

\section{Author Contributions}

DRM was involved in the design, experimental execution, laboratory analysis, generation of the figures, tables and graphs, and manuscript preparation; $\mathrm{ACBF}$ was involved in the design, experimental execution and reviewed the manuscript draft; MS was involved in the experimental execution; GP was involved in Fabry Sanger sequencing; MLSP was involved in the NPC and Gaucher validation, by Sanger sequencing; IVDS was involved in the Gaucher validation by Sanger sequencing; CFMS was the clinician of the Service responsible for the patients following; UM was involved in the experimental design and manuscript review; RG was involved in the manuscript review; All authors reviewed and commented on the manuscript during its drafting and approved the final version.

\section{References}

Adzhubei IA, Schmidt S, Peshkin L, Ramensky VE, Gerasimova A, Bork P, Kondrashov AS and Sunyaev SR (2010) A method and server for predicting damaging missense mutations. Nat Methods 7:248-249.

Bhattacharjee A, Sokolsky T, Wyman S, Reese MG, Puffenberger E, Strauss K, Morton H, Parad RB and Naylor EW (2015) Development of DNA confirmatory and high-risk diagnostic testing for newborns using targeted next-generation DNA sequencing. Genet Med 17:337-347.

Carter H, Douville C, Stenson PD, Cooper DN and Karchin R (2013) Identifying Mendelian disease genes with the Variant Effect Scoring Tool. BMC Genomics 14:S3.

Claes K and De Leener K (2014) Dealing with pseudogenes in molecular diagnostics in the next-generation sequencing era. Methods Mol Biol 11671:303-315.

Desmet FO, Hamroun D, Lalande M, Collod-Béroud G, Claustres M and Béroud C (2009) Human Splicing Finder: An online bioinformatics tool to predict splicing signals. Nucleic Acids Res 37:e67.

Douville C, Masica DL, Stenson PD, Cooper DN, Gygax D, Kim R, Ryan M and Karchin R (2016) Assessing the pathogenicity of insertion and deletion variants with the Variant Effect Scoring Tool (VEST-indel). Hum Mutat 37:28-35.

Fernandez-Marmiesse A, Morey M, Pineda M, Eiris J, Couce ML, Castro-Gago M, Fraga JM, Lacerda L, Gouveia S, PérezPoyato MS et al. (2014) Assessment of a targeted resequencing assay as a support tool in the diagnosis of lysosomal storage disorders. Orphanet J Rare Dis 9:59.

Filocamo M and Morrone A (2011) Lysosomal storage disorders: Molecular basis and laboratory testing. Hum Genomics 5:156-169.

Franco JF, Soares DC, Torres LC, Leal GN, Cunha MT, Honjo RS, Bertola DR and Kim CA (2016) Impact of early enzyme-replacement therapy for mucopolysaccharidosis VI: Results of a long-term follow-up of Brazilian siblings. Genet Mol Res 15:7850.

Gargis AS, Kalman L, Berry MW, Bick DP, Dimmock DP, Hambuch T, Lu F, Lyon E, Voelkerding KV, Zehnbauer BA et al. (2012) Assuring the quality of next-generation sequencing in clinical laboratory practice. Nat Biotechnol 30:1033-1036.

Giugliani R, Federhen A, Vairo F, Vanzella C, Pasqualim G, Silva LMR, Giugliani L, Boer APK, Souza CFM, Matte U et al. (2016) Emerging drugs for the treatment of mucopolysaccharidoses. Expert Opin on Emerg Drugs 21:9-26.

Kumar P, Henikoff S and Ng PC (2009) Predicting the effects of coding non-synonymous variants on protein function using the SIFT algorithm. Nat Protoc 4:1073-1081.

Lévesque S, Auray-Blais C, Gravel E, Boutin M, DempseyNunez L, Jacques PE, Chenier S, Larue S, Rioux MF, AlHertani W et al. (2016) Diagnosis of late-onset Pompe disease and other muscle disorders by next-generation sequencing. Orphanet J of Rare Dis 11:1-10.

Martins AM, Kyosen SO, Garrote J, Marques FM, Guilhem JG, Macedo E, Sobral Neto J and Ura S (2013) Demographic characterization of Brazilian patients enrolled in the Fabry Registry. Genet Mol Res 12:136-142.

McLaren W, Gil L, Hunt SE, Riat HS, Ritchie GR, Thormann A, Flicek P and Cunningham F (2016) The Ensembl Variant Effect Predictor. Genome Biol 17:122.

Miller SA, Dykes DD and Polesky HF (1988) A simple salting out procedure for extracting DNA from human nucleated cells. Nucleic Acids Res 16:1215.

Muenzer J (2014) Early initiation of enzyme replacement therapy for the mucopolysaccharidoses. Mol Genet Metab 111:63-72.

Rehm HL, Bale SJ, Bayrak-Toydemir P, Berg JS, Brown KK, Deignan JL, Friez MJ, Funke BH, Hegde MR, Lyon E et al. (2013) ACMG Clinical Laboratory Standards for Next-Generation Sequencing. Genet Med 5:733-747.

Richards S, Aziz N, Bale S, Bick D, Das S, Gastier-Foster J, Grody WW, Hegde M, Lyon E, Spector E et al. (2015) Standards and guideline for the interpretation of sequence variants: A joint consensus recommendation of the American College of Medical Genetics and Genomics and the Association of Molecular Pathology. Genet Med 17:405-424. 
Robinson JT, Thorvaldsdóttir H, Winckler W, Guttman M, Lander ES, Getz G and Mesirov JP (2011) Integrative Genomics Viewer. Nature Biotechnology 29:24-26.

Schwarz JM, Cooper DN, Schuelke M and Seelow D (2014) MutationTaster2: Mutation prediction for the deep-sequencing age. Nat Methods 11:361-362.

Sim NL, Kumar P, Hu J, Henikoff S, Schneider G and Ng PC (2012) SIFT web server: Predicting effects of amino acid substitutions on proteins. Nucleic Acids Res 40:452-457.

Tajima G, Sakura N, Kosuga M, Okuyama T and Kobayashi M (2013) Effects of idursulfase enzyme replacement therapy for Mucopolysaccharidosis type II when started in early infancy: Comparison in two siblings. Mol Genet Metab 108:172-177.

Tayoun ANA, Tunkey CD, Pugh TJ, Ross T, Shah M, Lee CC, Harkins TT, Wells WA, Tafe LJ, Amos CI et al. (2013) A comprehensive assay for CFTR mutational analysis using next-generation sequencing. Clin Chem 59:1481-1488.

Vieira T, Schwartz I, Muñoz V, Pinto L, Steiner C, Ribeiro M, Boy R, Ferraz V, de Paula A, Kim C et al. (2008) Mucopolysaccharidoses in Brazil: What happens from birth to biochemical diagnosis? Am J Med Genet A 146A:1741-1747.

Wang RY, Bodamer OA, Watson MS, Wilcox WR and ACMG Work Group on Diagnostic Confirmation of Lysosomal Storage Diseases (2011) Lysosomal storage diseases: Diagnostic confirmation and management of presymptomatic individuals. Genet Med 13:457-484.

Wood T, Basehore M, Jones J, Friez M, Cathey S and Pollard L (2013) Development of a next generation sequencing panel for lysosomal storage disorders. Mol Genet Metab 108:S100

\section{Supplementary material}

The following online material is available for this article:

Figure S1 - Relative Depth of Coverage, Panel A.

Figure S2 - Relative Depth of Coverage, Panel B.

Associate Editor: Mariluce Riegel

License information: This is an open-access article distributed under the terms of the Creative Commons Attribution License (type CC-BY), which permits unrestricted use, distribution and reproduction in any medium, provided the original article is properly cited. 